\title{
Integrated Lithium Niobate Acousto-optic Frequency Shifter
}

\author{
Linbo Shao ${ }^{1}$, Neil Sinclair ${ }^{1,2}$, James Leatham ${ }^{3}$, Yaowen $\mathrm{Hu}^{1}$, Mengjie $\mathrm{Yu}^{1}$, \\ Terry Turpin $^{4}$, Devon Crowe ${ }^{3}$, Marko Loncar ${ }^{1}$ \\ ${ }^{1}$ John A. Paulson School of Engineering and Applied Sciences, Harvard University, 29 Oxford Street, Cambridge, MA 02138, USA \\ ${ }^{2}$ Division of Physics, Mathematics and Astronomy, and Alliance for Quantum Technologies (AQT), California Institute of Technology, 1200 E. \\ California Blvd., Pasadena, California 91125, USA \\ ${ }^{3}$ Raytheon Space \& Airborne Systems, 2000 E. El Segundo Ave., El Segundo, CA 90245, USA and \\ ${ }^{4} 9918$ Evergreen Ave., Columbia, MD 21046, USA \\ shaolb@seas.harvard.edu; loncar@seas.harvard.edu
}

\begin{abstract}
We demonstrate a 3-GHz acousto-optic frequency shifter on a thin-film lithium niobate platform, featuring over $30 \mathrm{~dB}$ carrier suppression. The active frequency shifting loop generates a frequency comb with 200 lines over $5 \mathrm{~nm}$ optical bandwidth. @ 2020 The Author(s)
\end{abstract}

\section{Introduction}

Acousto-optic devices [1] that provide optical frequency shifting have various applications from heterodyne detection to optical frequency comb generation. Commercially available acousto-optic modulators utilize bulk acoustic modes and thus operate at low frequencies (at $10-100 \mathrm{~s}$ of $\mathrm{MHz}$ ) and require up to tens of Watts radio frequency (RF) powers. An alternative approach utilizes surface acoustic waves to deflect light confined by an ion diffused layer [2]. However, relatively large optical mode size of such an optical waveguide (a few microns) limits the interactions with sub-micronwavelength $\mathrm{GHz}$ acoustic waves. Electromechanical Brillouin scattering has been utilized to achieve frequency shifts exceeding $10 \mathrm{GHz}[3]$ but this approach suffers from low efficiencies of $\sim 10^{-5}$ and sub-optimal carrier suppression. Here, we demonstrate a 3-GHz integrated acousto-optic frequency shifter (AOFS) on the thin-film lithium niobate (LN) platform. In our approach, the traveling acoustic wave deflects $1.5-\mu \mathrm{m}$ wavelength optical beam at the Bragg angle of 18 degrees resulting in the carrier suppression $>30 \mathrm{~dB}$.

\section{Device design}

Our integrated AOFSs are fabricated using an 800-nm-thick X-cut LN thin film on a 2 - $\mu$ m-thick silicon dioxide layer on a silicon substrate (Fig. 1). The LN thin film confines both 1.5- $\mu \mathrm{m}$-in-wavelength light and 3-GHz acoustic waves. Our AOFSs are fully supported by the substrate, thereby providing improved robustness than suspended acousto-optic devices. The traveling acoustic wave deflects the input light and shifts its optical frequency. The phase matching condition (Fig. 1(a) inset) determines the optimum (Bragg) angle $\theta_{B}$ of the input light beam, given by $\operatorname{Sin} \theta_{B}=\frac{K}{2 k}=$ $\frac{\lambda}{2 \Lambda n_{\mathrm{eff}}}$, where the optical wavenumber $k=2 \pi n_{\mathrm{eff}} / \lambda, \mathrm{n}_{\mathrm{eff}}=2.0$ is the optical mode refractive index, and $\lambda=1.5 \mu \mathrm{m}$ is the optical wavelength in vacuum. The acoustic wavenumber $K=2 \pi / \Lambda$, where $\Lambda=1.2 \mu \mathrm{m}$ is the wavelength of an around 3-GHz acoustic wave in the LN thin film. These parameters result in the Bragg angle of $\operatorname{Sin} \theta_{B}=0.3$ $\left(\theta_{B} \sim 18\right.$ degrees). Our device can be configured for either anti-Stokes (towards higher) or Stokes (towards lower) frequency shifts.

\section{Experimental characterization}

We characterize our LN AOFS in the anti-Stokes configurations (Fig. 2). The optical spectra of the deflected light are measured by heterodyne detection using a second laser, which is a few gigahertz red-detuned from the input laser (Fig. 2(a)). The IDT is driven at its most efficient frequency of $2.89 \mathrm{GHz}$ with microwave power of $15 \mathrm{dBm}$. The deflected light is blue-shifted by $2.89 \mathrm{GHz}$ and exhibits a 33-dB carrier suppression (Fig. 2(c)). The other optical sideband with opposite frequency shifting is not observed above the noise floor, and the sideband suppression ratio thus exceeds 40 dB. A $30 \mathrm{dBm}$ maximum driving microwave power results in an on-chip deflection efficiency of $3.5 \%$ (Fig. 2(d)). The optical and microwave bandwidths are $14 \mathrm{~nm}$ and $70 \mathrm{MHz}$, respectively (Figs. 2(e) and 2(f)). Furthermore, we were able to generate an optical frequency comb with $2.9 \mathrm{GHz}$ line spacing using the active acousto-optic frequency shifting loop (Fig. 3). More than 200 lines over a $5 \mathrm{~nm}$ optical bandwidth are observed.

\section{Conclusions and Outlook}

Our integrated AOFS features strong carrier and side-band suppression, are competitive with the state-of-the-art discrete bulk acousto-optic devices. We believe that our acousto-optic platform will find applications in on-chip optical routers, scanners [4], isolators [5], microwave spectrum analyzers [6], and carrier-envelope phase stabilizers [7]. 

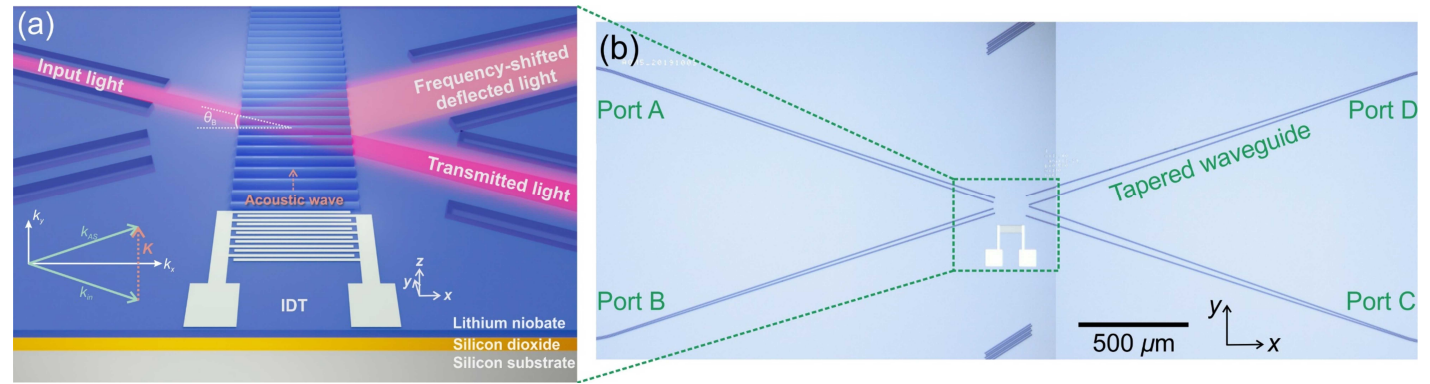

Fig. 1 (a) Schematic of the Thin-film LN AOFS. (b) Optical microscope image of the fabricated device.

(a)

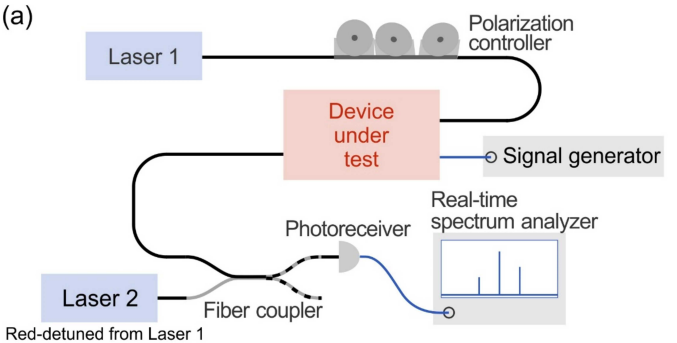

Red-detuned from Laser 1

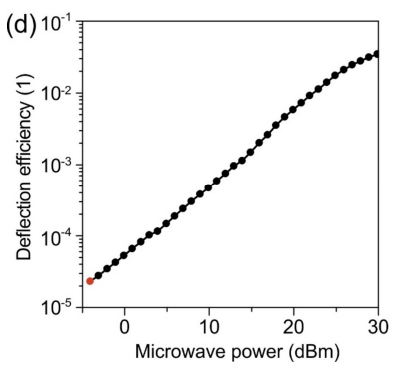

(b)

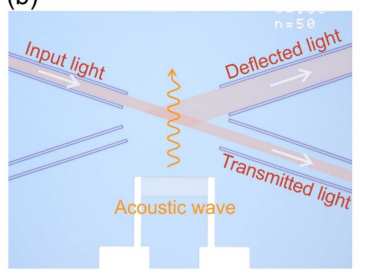

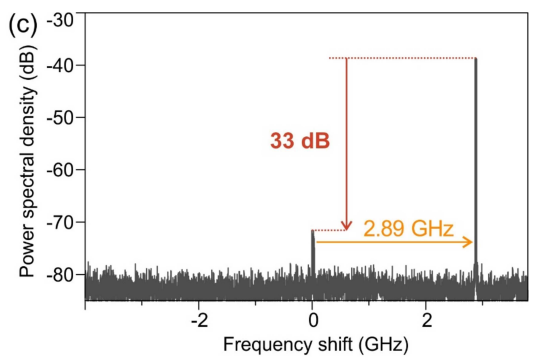
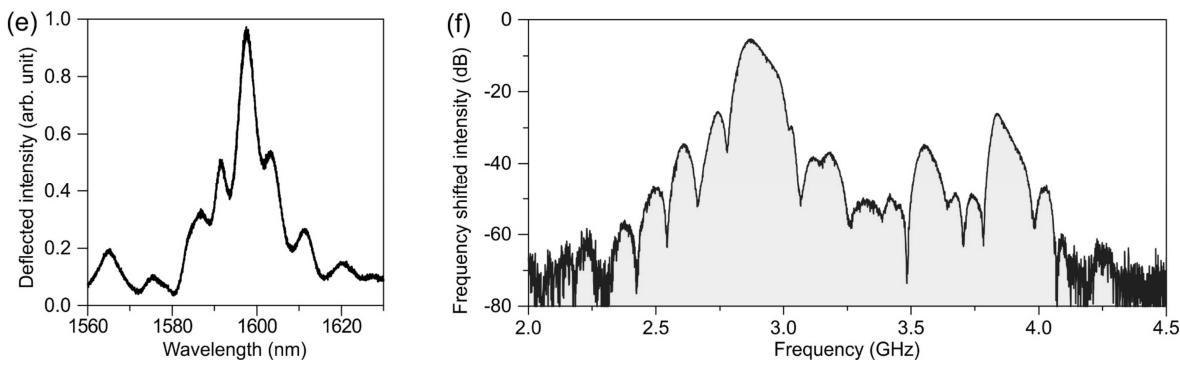

Fig. 2 (a) Experimental setup of the heterodyne detection. (b)(c) anti-Stokes frequency shifting configuration and the spectrum of the deflected light. (d) Efficiency of the frequency shifter for different microwave driving powers. (e) Deflected optical power for different wavelengths of the optical signal used. (f) Optical intensity of the frequency-shifted light vs. the microwave frequency, measured by the homodyne detection.

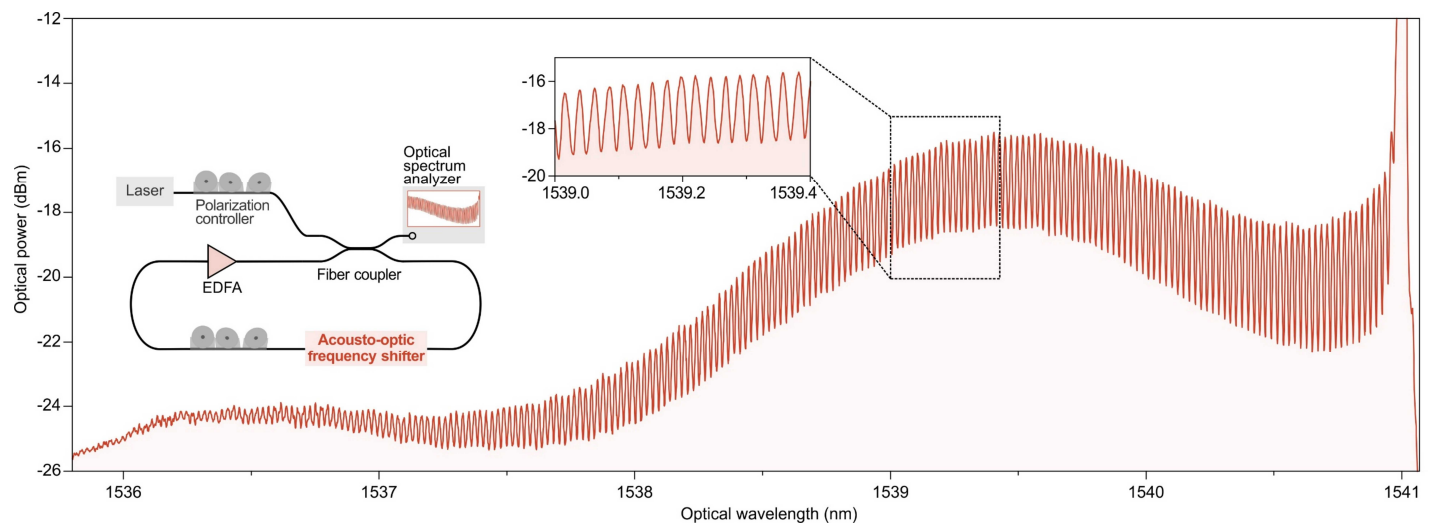

Fig. 4 Optical frequency comb generation using an active frequency shifting loop.

\section{Reference}

[1] N. Savage, "Acousto-optic devices," Nat. Photonics 4, 728-729 (2010).

[2] Z. Y. Cheng, and C. S. Tsai, "Baseband integrated acousto-optic frequency shifter," Appl. Phys. Lett. 60, 12-14 (1992).

[3] H. Li, et al., "Electromechanical Brillouin scattering in integrated planar photonics," APL Photonics 4, 080802 (2019).

[4] B. E. A. Saleh, and M. C. Teich, "Ch. 20 Acousto-optics," in Fundamentals of photonics(John Wiley \& Sons, 1991).

[5] Z. Yu, and S. Fan, "Complete optical isolation created by indirect interband photonic transitions," Nat. Photonics 3, 91-94 (2009).

[6] T. M. Turpin, "Spectrum analysis using optical processing," Proc. IEEE 69, 79-92 (1981).

[7] S. Koke, et al., "Direct frequency comb synthesis with arbitrary offset and shot-noise-limited phase noise," Nat. Photonics 4, 462-465 (2010).

Disclaimer: This document does not contain technology or Technical Data controlled under either the U.S. International Traffic in Arms Regulations or the U.S. Export Administration Regulations. 